\title{
Comparison of different phase retrieval algorithms
}

\author{
Rolf KAUFMANN ${ }^{a}$, Mathieu PLAMONDON ${ }^{a}$, Jürgen $\mathrm{HOFMANN}^{a}$, Antonia NEELS ${ }^{a}$ \\ ${ }^{a}$ Empa, Swiss Federal Laboratories for Materials Science and Technology, \\ Überlandstrasse 129, 8600 Dübendorf, Switzerland
}

\begin{abstract}
$\mathrm{X}$-ray phase contrast imaging is attracting more and more interest. Since the phase cannot be measured directly an indirect method using e.g. a grating interferometer has to be applied. This contribution compares three different approaches to calculate the phase from Talbot-Lau interferometer measurements using a phase-stepping approach. Besides the usually applied Fourier coefficient method also a linear fitting technique and Taylor series expansion method are applied and compared.
\end{abstract}

\section{INTRODUCTION}

Grating based phase contrast imaging is an emerging X-ray technology for low absorbing or for structured materials. For this measurement a Talbot-Lau interferometer with three gratings and a phase stepping procedure was used to extract absorption-, differential phase- and dark-field images simultaneously. ${ }^{1,2}$ Another possible implementation of this method can be found in reference. ${ }^{3,4}$

\section{MEASUREMENT SETUP}

The measurement system consisted of a three-grating Talbot-Lau interferometer (Figure 1) with a design energy of $35 \mathrm{keV}$ at the Empa Center of X-ray Analytics ${ }^{5}$. A schematic of this setup is shown in Figure 2. The gratings $G_{1}$ and $G_{2}$ have a field of view of $50 \times 50 \mathrm{~mm}^{2}$, whereas the grating $G_{0}$ is much smaller and sits directly on top of the exit window of the source. The gratings have the following periodicities: $p_{0}=30 \mu \mathrm{m}, p_{1}=2.92 \mu \mathrm{m}$ and $p_{2}=$ $3.24 \mu \mathrm{m}$. The gratings $G_{1}$ and $G_{2}$ were purchased from Microworks, Germany, and $G_{0}$ from CSEM, Switzerland. The source was a closed macro-focus tube from Varian (HPX-160/11) and the detector a CMOS imager with $49.5 \mu \mathrm{m}$ pixel size and a GOS scintillator (Teledyne Dalsa Shad-o-Box 6K HS DRZ). The sample stage is normally positioned right in front of the grating $G_{1}$. This configuration gives the highest phase-sensitivity. If more spatial

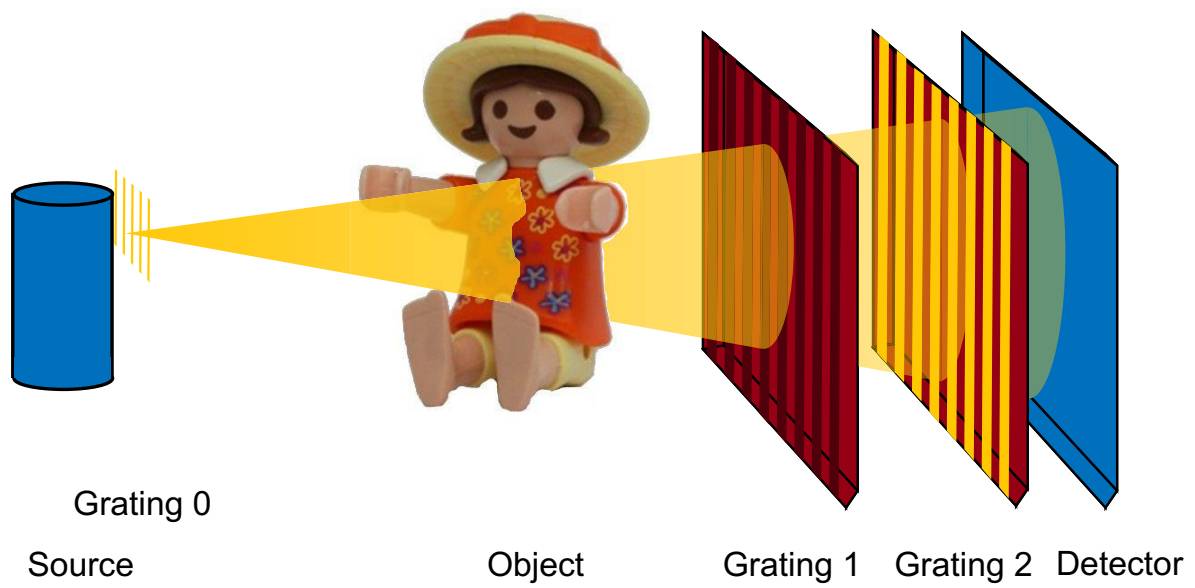

Figure 1. Schematic principle of a Talbot-Lau interferometer. 


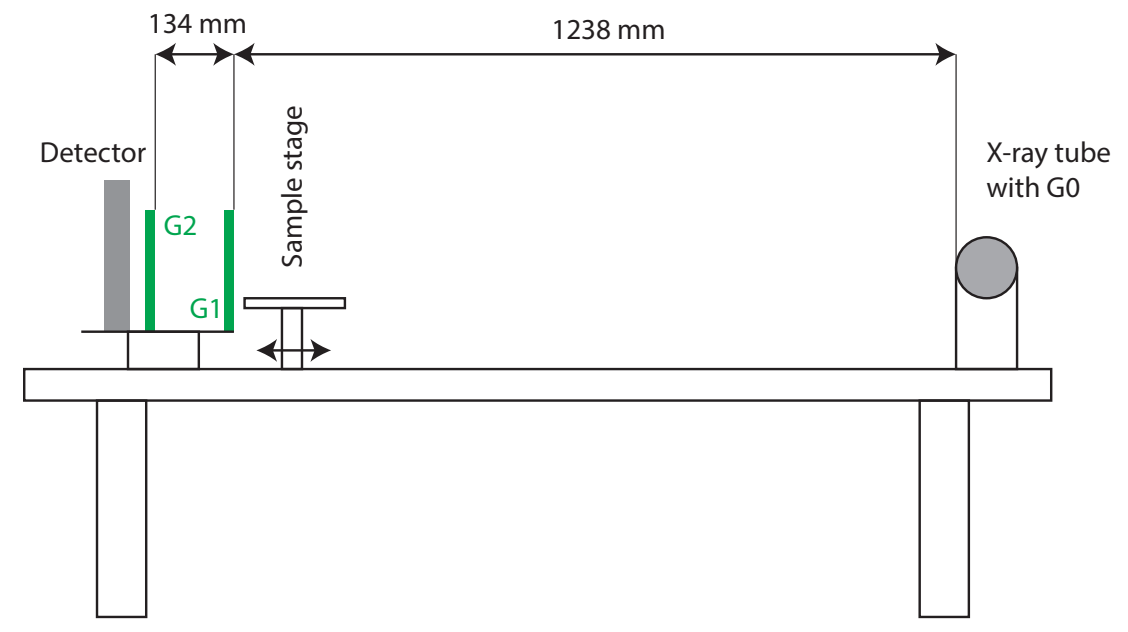

Figure 2. Sketch of the asymmetric Talbot-Lau interferometer used for this analysis. The source grating $G_{0}$ is mounted inside the source.

resolution is required it can also be moved closer to the source and hence a higher magnification is possible, but with a corresponding loss in phase-sensitivity. Two photographs of the system are shown in Figure 3 and it is also described further in reference ${ }^{6}$.

Compared to synchrotron based implementations of such interferometers ${ }^{7-10}$ the flux is always limited by the power of the X-ray source and therefore measurements are quite slow. Even industrial macro-focus sources are not powerful enough to image dynamic processes which happen within fractions of seconds. For such measurements a synchrotron source is compulsory. But for static or slowly changing systems laboratory implementations of this interferometer are completely competitive.

\section{FOURIER DECOMPOSITION}

The extraction of the three images requires a processing step (Figure 4). The usually employed method is a Fourier decomposition using a fast-Fourier transform (FFT). ${ }^{11}$

The Fourier decomposition assumes that the phase stepping curve is sine-like, which is a sound assumption for rectangular gratings of a reasonable quality. But this method also has its drawbacks. It requires that the phase stepping corresponds to one or several full periods of the grating that is analysed. But a phase stepping over e.g only half a period will not work. It is also required that the individual phase-steps are equally distributed over the whole period.

This article examines two other possible phase retrieval algorithms which also work with phase stepping curves outside full $2 \pi$ periods. These two approaches are compared to the standard Fourier decomposition and advantages and disadvantages are explained.

In all cases the phase stepping curve is approximated by a sine or cosine function

$$
S=M+A \sin (x+\phi) \quad,
$$

where $M$ is the mean value of the phase stepping curve, $A$ its amplitude and $\phi$ the phase value. In a phase stepping measurement the signal $S_{i}$ is measured at discrete phase-steps $x_{i}$. In the classical Fourier analysis the 


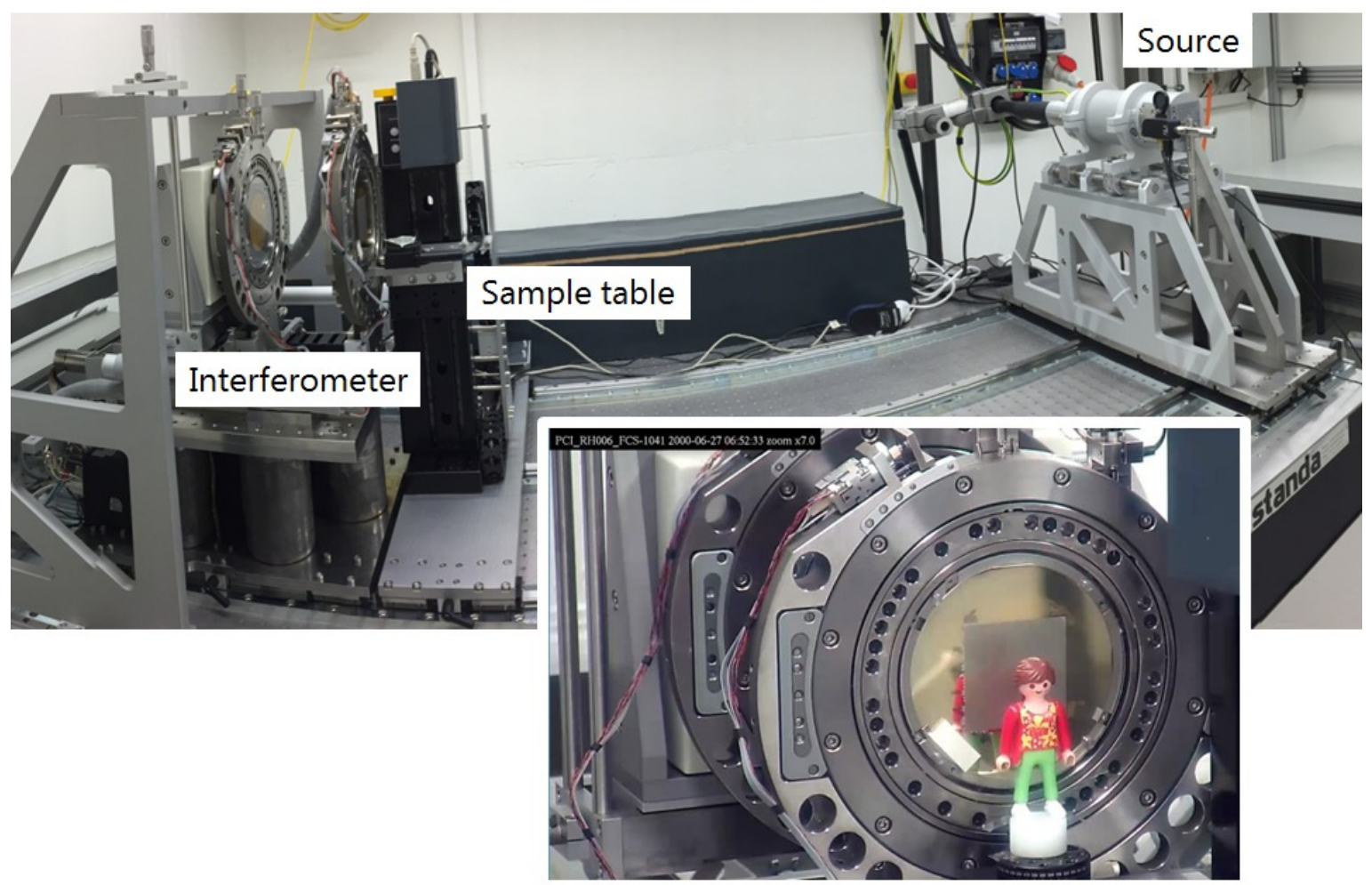

Figure 3. Sideview of the setup (top) and the sample in front of the gratings seen from the source.

parameters $M, A$ and $\phi$ are extracted from the first two coefficients of the Fourier transform of $S_{i}$

$$
\begin{aligned}
M & =\left|F_{0}\left(S_{i}\right)\right| \\
A & =2 \cdot\left|F_{1}\left(S_{i}\right)\right| \\
\phi & =\arg \left(F_{1}\left(S_{i}\right)\right)
\end{aligned}
$$

where $F_{0}$ and $F_{1}$ are the first and second complex Fourier coefficients.

\section{LINEARISED FIT}

The phase stepping curves can be determined by solving the overdetermined system of equations in (1). This can be performed directly by applying non-linear square fit (NLS) methods like the Levenberg-Marquardt ${ }^{12}$ algorithm. NLS methods imply iterative solvers which do not guarantee that the retrieved solution converge to a global minimum. ${ }^{13}$ But equation (1) can easily be transformed such that linear square fit (LLS) methods can be applied.

The linearised equation is

$$
S=M+c_{1} \sin (x)+c_{2} \cos (x)
$$

where the amplitude $A$ can be determined as

$$
A=\sqrt{c_{1}^{2}+c_{2}^{2}}
$$

and the phase $\phi$

$$
\phi=\arctan \left(\frac{c_{1}}{c_{2}}\right) \quad .
$$




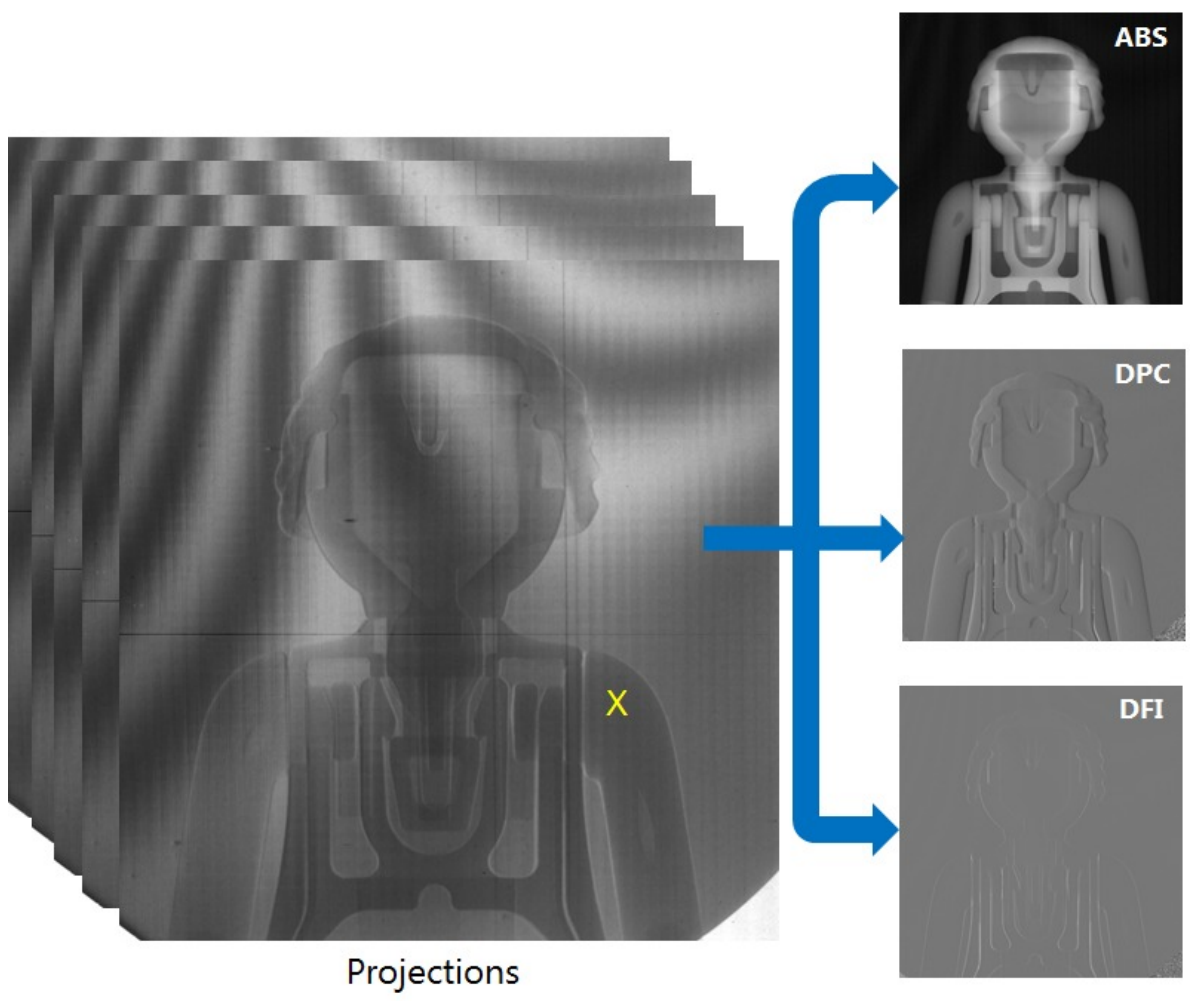

Figure 4. A series if images is acquired where one of the three gratings is moved over one period. From this series the differential phase-, darkfield- and absorption images are calculated. The pixel in the shoulder of the figure marked with a yellow $\mathrm{X}$ was used for the further analysis.

In equation (5) all parameters $\left(c_{1}, c_{2}\right.$ and $\left.M\right)$ are linear in respect to the LLS problem. Thus we have to solve the minimisation problem

$$
C^{\prime}=\arg \underset{C}{\min } O(C)
$$

where the objective function $O(C)$ is

$$
O(C)=\|S-X C\|^{2}
$$

with

$$
S=\left(\begin{array}{r}
S_{1} \\
S_{2} \\
S_{3} \\
\vdots \\
S_{m}
\end{array}\right), \quad X=\left(\begin{array}{rrr}
1 & \sin \left(x_{1}\right) & \cos \left(x_{1}\right) \\
1 & \sin \left(x_{2}\right) & \cos \left(x_{2}\right) \\
1 & \sin \left(x_{3}\right) & \cos \left(x_{3}\right) \\
\vdots & \vdots & \vdots \\
1 & \sin \left(x_{m}\right) & \cos \left(x_{m}\right)
\end{array}\right), \quad C=\left(\begin{array}{r}
M \\
c_{1} \\
c_{2}
\end{array}\right), \quad \text { phase step } m: x_{m} \in[-\pi,+\pi]
$$

In this paper the open source library ArrayFire ${ }^{14}$ is used to solve the LLS problem. ArrayFire itself utilises LAPACK $^{15}$ for solving dense linear system equations.

\section{TAYLOR SERIES EXPANSION}

The sine or cosine function can also be developed in a Taylor series which is linear in the argument $\phi^{16}$ :

$$
S_{i}=M+A \sin \left(x_{i}-\phi\right)+n_{i} \approx M+A \sin \left(x_{i}\right)-A \phi \cos \left(x_{i}\right)+n_{i}
$$



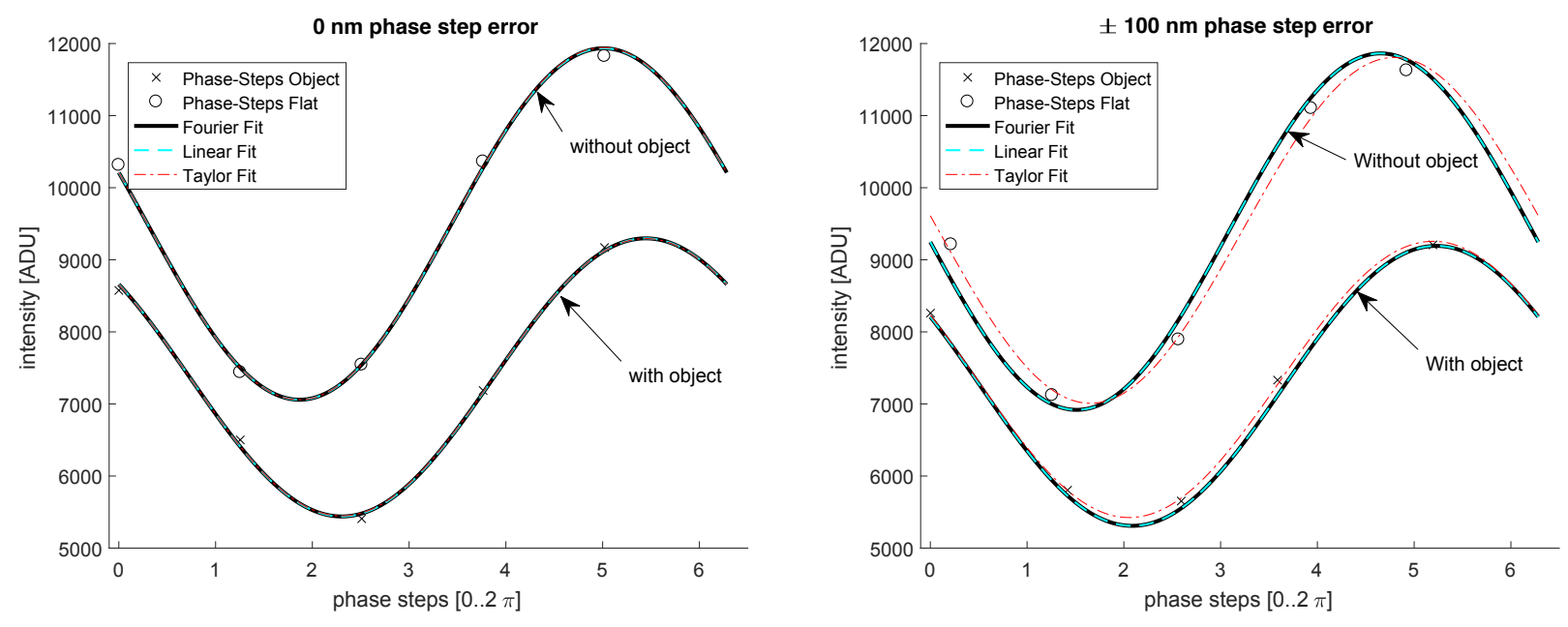

Figure 5. Phase stepping curves for 5 phase steps of all three methods, without (left) and with $\pm 100 \mathrm{~nm}$ random phasestepping errors (right).

where $n_{i}$ is the the noise for each phase step $i$.

$A$ and $A \phi$ can be expressed as linear combinations of the sampled values $S_{i}$ :

$$
A=\sum a_{i} S_{i} \quad A \phi=\sum b_{i} S_{i}
$$

where $a_{i}$ and $b_{i}$ are determined by minimising the impact of the noise $n_{i}$ with the constraint of having unbiased estimators.

\section{COMPARISON}

Since the phase stepping only spans a few micrometers it needs a very precise motor. Piezo-Motors are normally employed since they offer such repetition accuracies, but are generally quite expensive. If a motor with less precision could be employed, e.g. a stepping motor, it would have a beneficial influence on the cost of the interferometer system.

For this reason, a random error of up to $\pm 100 \mathrm{~nm}$ was artificially added on the phase-stepping system to emulate a phase-stepping motor with less precision. This was done with a box-distributed random error generator, which added the errors to the precise phase-stepping points before sending them to the motor. The resulting phase-stepping curves for five steps are shown in Figure 5.

As can be seen all three methods give very similar results for one measurement setting. The amplitude $A$ and mean values $M$ of the fitted curves also matched approximately within $2 \%$ independent of the number of phase-steps and phase errors. But the phase values $\Phi$ varied up to $30 \%$, depending on the number of phase steps and the introduced error. This also corresponds to the findings in reference ${ }^{17}$. But for each number of phase steps and each error value all three methods lead to similar results.

For accurate quantitative phase measurements it is therefore necessary to use a large number of phase-steps and a very precise phase stepping-motor. But for only qualitative comparisons a phase stepping procedure with less phase-steps can be used provided that all measurements are made with the same number of phase-steps. All three presented methods can be used to retrieve mean, amplitude and phase with similar results. 


\section{RESULTS}

All three methods lead to very similar results for the same settings. But especially for the retrieved phase values, there are significant differences depending on the number of phase steps and the stepping errors. This differences can mount up to $30 \%$ or more. For quantitative phase measurements a large number of phase steps (more than 10) should be considered and a precise motor should be employed (accuracy better than $5 \mathrm{~nm}$ ).

The non-Fourier based phase-retrieval methods have the advantage that they also work with non- $2 \pi$ coverage and with non-equidistant phase steps. With parallel computing (using e.g. software package cuSOLVER ${ }^{18}$ ) they can be made as fast as the Fourier method based on FFT.

Furthermore, they can even be developed further to partially correct some of the shown errors. Especially the Taylor method can be used to compensate for noise influences and can even estimate position inaccuracies of the individual phase steps. This correction is currently under development and will be implemented in the future.

\section{DISCUSSION AND OUTLOOK}

All measurements were taken with one setup at Empa. Even though another setup may show slightly different absolute phase values due to different X-ray spectra or grating materials (beam hardening), there is no reason to believe that the three presented methods will not lead to comparable results, since they don't depend on any assumption concerning the setup (as long as rectangular gratings are used).

A theoretical analysis of measurement uncertainties in X-ray grating interferometry is e.g. given in references $^{17,19}$. In the current study no estimation of the absolute phase-measurement uncertainty was made since the material of the investigated object was unknown. The goal was more to compare the performance of three different phase retrieval algorithms. All of them lead to similar results concerning the phase values.

But the non-Fourier based methods have the advantage that the phase-stepping does not have to cover the full period and that can cope better with non-equally distributed support-points. This makes it possible to employ less accurate phase-stepping motors which come at a much lower price than e.g. Piezo based motors.

In the future the Taylor based method will be expanded to include also active corrections of phase-stepping errors to make it even less dependent on accurate phase-stepping motors.

\section{REFERENCES}

[1] Momose, A., Takano, H., Wu, Y., and Hoshino, M., "Recent advance in grating-based x-ray phase tomography," SPIE Proc. 10391, 0 (2017).

[2] Momose, A., Kuwabara, H., , and Yashiro, W., "X-ray phase imaging using lau effect," Appl. Phys. Express 4, 066603 (2011).

[3] Olivo, A. and Speller, R., "A coded-aperture technique allowing x-ray phase contrast imaging with conventional sources," 91, 074106.

[4] Olivo, A., Diemoz, P. C., Endrizzi, M., Hagen, C. K., Vittoria, F. A., Astolfo, A., Munro, P. R. T., Modregger, P., Kallon, G. K. N., Basta, D., Buchanan, I., Jones, C. M., and Zamir, A., "Fast and low-dose phase contrast ct with non-microfocal laboratory x-ray sources," SPIE Proc. 10391, 0 (2017).

[5] www.empa.ch/X-ray.

[6] Gusenbauer, C., Reiter, M., Plank, B., Senck, S., Hannesschläger, C., Renner, S., Kaufmann, R., and Kastner, J., "Multi-modal talbot-lau grating interferometer xct data for the characterization of carbon fibre reinforced polymers with metal components," Proc. iCT2017 www.ndt.net/?id=20807 (2017).

[7] Hipp, A. C., Vogelgesang, M., Mohr, J., and Kopmann, A., "High-resolution grating interferometer for phase-contrast imaging at petra iii," SPIE Proc. 10391, 0 (2017).

[8] Yang, F., Griffa, M., Hipp, A., Derluyn, H., Moonen, P., Kaufmann, R., Boone, M. N., Beckmann, F., and Lura, P., "Advancing the visualization of pure water transport in porous materials by fast, talbot interferometry-based multi-contrast x-ray micro-tomography," SPIE Proc. 9967, 99670L-6 (2016).

[9] Schulz, G., "Grating-based tomography applications in biomedical engineering," SPIE Proc. 10391, 0 (2017).

[10] Thalmann, P., Bikis, C., Hipp, A., Müller, B., Hieber, S. E., and Schulz, G., "Single and double grating-based x-ray microtomography using synchrotron radiation," Applied Physics Letters. 110, 061103 (2017). 
[11] Pfeiffer, F., Weitkamp, T., Bunk, O., and David, C., "Phase retrieval and differential phase-contrast imaging with low-brilliance x-ray sources," Nature Physics 2(4), 258 (2006).

[12] Levenberg, K., "A method for the solution of certain non-linear problems in least squares," Quarterly of Applied Mathematics 2, 164-168 (1944).

[13] Agarwal, S., Mierle, K., and Others, "Ceres solver." http://ceres-solver.org (2016).

[14] Yalamanchili, P., Arshad, U., Mohammed, Z., Entschev, P. G. P., Kloppenborg, B., Malcolm, J., and Melonakos, J., [ArrayFire - A high performance software library for parallel computing with an easy-to-use API], Atlanta: AccelerEyes.

[15] "Lapack linear algebra package." https://github.com/Reference-LAPACK (2017).

[16] Stern, E. and Cleland, W., "Signal processing considerations for liquid ionization calorimeters in a high rate environment," Nucl. Instrum. Meth. Phys. Res. A 338, 467-497 (1994).

[17] Revol, V., Kottler, C., Kaufmann, R., Straumann, U., and Urban, C., "Noise analysis of grating-based x-ray differential phase contrast imaging," Review of Scientific Instruments 81, 073709 (2010).

[18] Eaton, J., "Parallel direct solvers with cusolver, batched qr." https://devblogs.nvidia.com/parallelforall/paralleldirect-solvers-with-cusolver-batched-qr/ (2015).

[19] Raupach, R. and Flohr, T. G., "Analytical evaluation of the signal and noise propagation in x-ray differential phase-contrast computed tomography," Phys. Med. Biol. 56, 2219 (2011). 